\title{
Spatial Color Histograms for Content-Based Image Retrieval
}

\author{
Aibing Rao, Rohini K. Srihari, Zhongfei Zhang \\ Center of Excellence for Document Analysis and Recognition \\ State University of New York At Buffalo \\ arao, rohini, zhongfei@ cedar.buffalo.edu
}

\begin{abstract}
Color histogram is an important technique for color image database indexing and retrieving. In this paper, traditional color histogram is modified to capture spatial layout information of each color and three types spatial color histograms are introduced: annular, angular and hybrid color histograms. Experiments show that with a proper trade-off between the granularity in color dimensions and that in spatial dimensions, the performance of these histograms outperforms the traditional color histogram and some existing histogram refinement such as color coherent vector.
\end{abstract}

Keywords: Color Histogram, Spatial Color Histogram, Content-Based Image Retrieval, Annular Color Histogram, Angular Color Histogram, Hybrid Color Histogram.

\section{Introduction and Related Works}

Color histogram is one of the most important techniques for content-based image retrieval[9] because of its efficiency and effectiveness. However, due to the statistical nature, color histogram can only index the content of images in a limited way. To make color histogram more effective for image indexing, spatial information should be considered[1, 4, 6]. In the following, new techniques are proposed to extend color histogram in one more dimension. In addition to the the statistics in the dimensions of a color space, the distribution state of each single color in the spatial dimension is also taken into account. The combination of the statistics in the color and the spatial layout is called as spatial color histogram.

Starting from a mathematical model on how to characterize the distribution state of a set of geometric points on a 2-D plane under constraints such as translation and rotation invariant, a concept of distribution density is introduced in section 2. In section 3, this concept is applied to images, and three types of densities: annular, angular and hybrid distribution density, are introduced. This density information is used to modify the traditional histogram to get the spatial color histogram of an image. The refined histogram is presented as a $M \times N$ matrix where $M$, the number of the rows, is the granularity of the histogram in the color dimension and $N$, the number of columns, is the granularity of the spatial color histogram in the spatial dimension. Implementation issues and experiments of the comparison among the newly proposed techniques, the traditional one and some existing histogram refinement technique such as color coherent vector[3] are presented in section 4.

There are several techniques proposed to integrate spatial information with color histograms. Hsu et al. [1] integrates spatial information with color histograms by first selecting a set of representative colors and then analyzing the spatial information of the selected colors using maximum entropy quantization with event covering method. Stricker and Dimai [8] partition an image into 5 partially overlapping, fuzzy regions, extract the first three moments of the color distribution for each region, and then organize them into a feature vector of small dimension. Smith and Chang [6] apply back-projection on binary color sets to extract color regions. Pass and Zabih [3] define the concept of color coherent vector $(C C V)$ and use it to split a color histogram vector into two parts: a coherent vector and a non-coherent vector. A pixel is called coherent if its connected component is large enough. A $C C V$ of an image is the histogram over all coherent pixels of the image. Later, Huang [2] proposes color correlogram for refining histogram.

The difference between the approaches developed in this paper and above works is that the importance of the spatial information is made equal to that of color, and the spatial and color contributions to a final histogram can be balanced via tuning the quantization parameters.

\section{Distribution Density of a Finite Set of Points on a 2-D Plane}

The mathematical model is stated informally as: Given a finite set of geometric points on a 2-D plane, how to characterize the "distribution state" of the set? How to define similarity of two such sets? This is perfect for modeling spatial color histogram. Traditional histogram is only the statistics of the amount of each color so that the distribution 
manner of each color on the image is neglected. General observations state that the "spatial distribution state" of each color in an image is also important to the content of the image. Intuitively, the color histogram is a statistics on "how much" of each color contributing to the final histogram of an image, while a spatial distribution state of each color is a statistics on "where and how" the color is distributed in the image. Given a color quantization, for each color bin, define all of the pixels whose colors are in the bin as histogram subset of the bin. In the following, a mathematical analysis of a histogram subset is conducted.

Given a 2-D plane associated with a coordinate system, let $\mathcal{S}=\left\{\left(x_{1}, y_{1}\right),\left(x_{2}, y_{2}\right), \ldots,\left(x_{n}, y_{n}\right)\right\}$ be a set of $n$ points on the plane and $x_{i}, y_{i}$ are $\mathrm{x}$-coordinate and y-coordinate of the $i^{t h}$ points. In the following, any superset of $\mathcal{S}$ is called a universe over $\mathcal{S}$.

Definition 1. Given a universe $\mathcal{U}$ over $\mathcal{S}$, a measure of the distribution state of $\mathcal{U}$ is a mapping $\mathcal{F}$ from all of the subsets of $\mathcal{U}$, denoted as $2^{\mathcal{U}}$, to some $k$-dimensional Euclidean space $\mathcal{R}^{k}$, i.e. $\mathcal{F}: 2^{\mathcal{U}} \longrightarrow \mathcal{R}^{k} . \mathcal{F}(\mathcal{S})$ is called the distribution density of $\mathcal{S}$ and $k$ is called the granularity.

From now on, for a given set $\mathcal{S},|\mathcal{S}|$ always refers to the cardinality of $\mathcal{S}$.

Example 1. $\mathcal{F}(\mathcal{S})=|\mathcal{S}|$, the cardinality of set $\mathcal{S}$ is a distribution density of $\mathcal{S}$ with granularity degree $\mathbf{1}$.

Example 2. Given any partition $\left\{\mathcal{U}_{i}, 1 \leq i \leq p\right\}$ of the universe $\mathcal{U}$, a density function $\mathcal{F}: 2^{\mathcal{U}} \longrightarrow \mathcal{R}^{p}$ can be defined as follows, for any $\mathcal{S} \in 2^{\mathcal{U}}, \mathcal{F}(\mathcal{S})=\left(\left|\mathcal{S}_{1}\right|,\left|\mathcal{S}_{2}\right|\right.$ $\left., \ldots,\left|\mathcal{S}_{p}\right|\right)$ with $\mathcal{S}_{i}=\mathcal{S} \cap \mathcal{U}_{i}$ for $i=1,2, \ldots, p . \mathcal{F}(\mathcal{S})$ is a distribution density of $\mathcal{S}$ with granularity degree $\mathbf{p}$.

The traditional color histogram vector is a special case. The universe $\mathcal{U}$ is the set of all the pixel positions of the image and the partition is based on the color quantization.

Any distance measure for Euclidean space can be used to define similarity measure of two subsets under a same universe with a distribution measure.

Definition 2. Notations are as above. Let $\mathcal{S}_{1}$ and $\mathcal{S}_{2}$ be two sets in universe $\mathcal{U}$ with distribution measure $\mathcal{F}$. Suppose $\mathcal{D}(\cdot, \cdot)$ is a distance for $\mathcal{R}^{k}$, then $\mathcal{D}\left(\mathcal{F}\left(\mathcal{S}_{1}\right), \mathcal{F}\left(\mathcal{S}_{2}\right)\right)$ is called the similarity distance between $\mathcal{S}_{1}$ and $\mathcal{S}_{2}$ with respect to $\mathcal{F}$.

Next, the concept is used to define spatial color histogram of images.

\section{Annular, Angular and Hybrid Histograms}

Let $\left(p_{i j}\right)_{C \times R}$ be an image of size $C \times R$ where $p_{i j}$ is the color of pixel $(i, j)$. Let $\mathcal{U}=\{(x, y) \mid 1 \leq x \leq R ; 1 \leq$ $y \leq C\}$. Suppose $B_{1}, B_{2}, \ldots, B_{M}$ are the blocks in the color space after quantization with $M$ color bins. Let $\mathcal{S}_{q}=$ $\left\{(x, y) \mid(x, y) \in \mathcal{U}, p_{x y} \in B_{q}\right\}$ for $1 \leq q \leq M$, then $\mathcal{U}=\bigcup_{q=1}^{M} \mathcal{S}_{q}$ is a partition of $\mathcal{U}$. Here each $\mathcal{S}_{q}$, called histogram subset of bin $B_{q}$, is the set of pixels whose color is in the $q^{t h}$ bin.

Next consider the histogram subset $\mathcal{S}_{q}$ as a geometric set on the $2-D$ plane for each color bin $B_{q}$. Let $C^{q}=\left(x^{q}, y^{q}\right)$ be the centroid of $\mathcal{S}_{q}$, where $x^{q}$ and $y^{q}$ are defined as

$$
x^{q}=\frac{1}{\left|\mathcal{S}_{q}\right|} \sum_{(x, y) \in \mathcal{S}_{q}} x ; \quad y^{q}=\frac{1}{\left|\mathcal{S}_{q}\right|} \sum_{(x, y) \in \mathcal{S}_{q}} y
$$

Let $r^{q}$ be the radius of $\mathcal{S}_{q}$ which is defined as

$$
r^{q}=\max _{(x, y) \in \mathcal{S}_{q}} \sqrt{\left(x-x^{q}\right)^{2}+\left(y-y^{q}\right)^{2}}
$$

Given a number $N$, uniformly divide the radius into $N$ buckets, then draw $N$ concentric circles with $C^{q}$ as the center and with $\frac{k r^{q}}{N}$ as the radius for each $1 \leq k \leq N$ to form $N$ annular regions. The intersections of $\mathcal{S}_{q}$ with each of the regions from the innermost to the out-most one, $R_{q 1}, R_{q 2}, \ldots, R_{q N}$, give a partition of $\mathcal{S}_{q}$.

Definition 3. Vector $\left(\left|R_{q 1}\right|,\left|R_{q 2}\right|, \ldots,\left|R_{q N}\right|\right.$ ) is called the annular distribution density of the set $\mathcal{S}_{q}$. $N$ is called the spatial granularity of the density. This is illustrated in Figure 1.

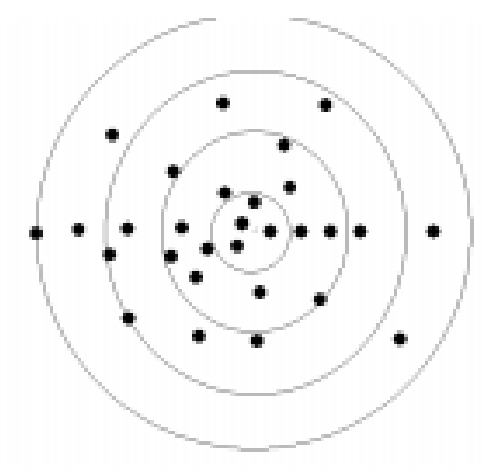

Figure 1. Annular distribution density vector is calculated by counting the number of points in each annular region. The vector is: $(4,11,9,5)$.

Set $A_{\alpha \beta}=\left|R_{\alpha \beta}\right|$ for $\alpha=1,2, \ldots, M$ and $\beta=1,2, \ldots, N$, then a $M \times N$ matrix $A=\left(A_{\alpha \beta}\right)_{M \times N}$ is a modification of the traditional histogram. This matrix is called the annular color histogram of the image. The simplest similarity distance is just the Euclidean distance by regarding the matrix as a 1-dimensional vector. Since the centroid and the annular partition of each histogram subset are translation and rotation invariant, so is the annular color histogram, implying that the histogram is tolerant to a small movement of a camera when images are taken.

Notice that when $N=1$, the matrix is degraded to the traditional histogram. 
Similar to the annular partition, angular partition introduces another type of spatial color histogram. In order to make angular partition also preserve the translation and rotation invariant, a starting direction of the partition is determined at first. Consider a coordinate system associated to $\mathcal{S}_{q}$ by translate the original coordinate system to the centroid $C^{q}=\left(x^{q}, y^{q}\right)$ of $\mathcal{S}_{q}$. For each point $(x, y) \in \mathcal{S}_{q}$, calculate the direction(principal angle) $\theta(x, y)$ of the point in the associated coordinate system via

$$
\theta(x, y)=\arctan \left(\frac{y-y^{q}}{x-x^{q}}\right) \pm \pi
$$

where,+- are to be selected depending on which quadrant the point is in. Then the average direction, denoted as $\Theta\left(\mathcal{S}_{q}\right)$, of the subset $\mathcal{S}_{q}$, called the principal direction of $\mathcal{S}_{q}$ is

$$
\Theta\left(\mathcal{S}_{q}\right)=\frac{1}{\left|\mathcal{S}_{q}\right|} \sum_{(x, y) \in \mathcal{S}_{q}} \theta(x, y)
$$

Notice that the principal direction of $\mathcal{S}_{q}$ is both translation and rotation invariant with respect to the original image. Now given a number $N$, for each histogram subset $\mathcal{S}_{q}$, staring from the principle direction $\Theta\left(\mathcal{S}_{q}\right)$, uniformly divide the unit circle centered at $C^{q}$ into $N$ arcs so that the plane is equally divided into $N$ fan-like domains, called angular regions, then starting from the principal direction, partition $\mathcal{S}_{q}$ by intersecting it with each of the angular regions counterclockwise to get: $R_{q 1}, R_{q 2}, \ldots, R_{q N}$.

Definition 4. With above angular partition, $\left(\left|R_{q 1}\right|, \mid\right.$ $\left.R_{q 2}|, \ldots,| R_{q N} \mid\right)$ is called the angular distribution density of the set $\mathcal{S}_{q}$. This scenario is illustrated in Figure 2.

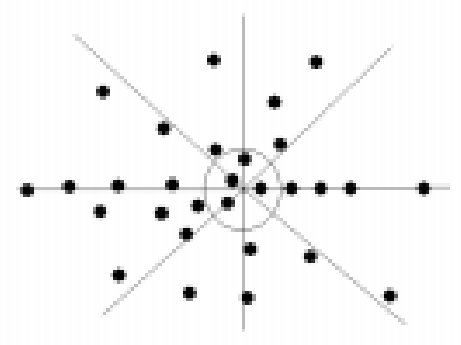

Figure 2. Angular distribution density vector is calculated by counter-clockwise counting the number of points in each angular region, starting from the principal direction. The vector is: $(5,4,2,6,7,1,3,1)$.

By combining above two approaches, a hybrid partition of $\mathcal{S}_{q}$ is proposed in Figure 3.
Definition 5. With the hybrid partition, the associated cardinality vector is called the hybrid distribution density of the set $\mathcal{S}_{q}$.

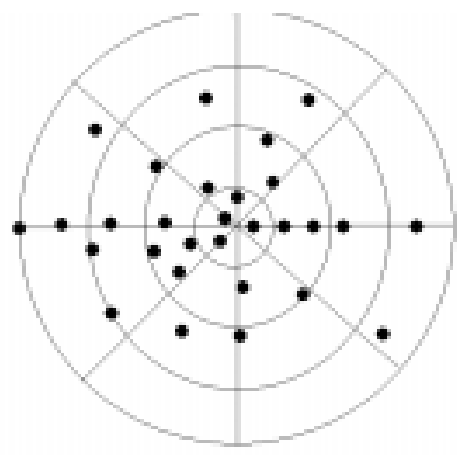

Figure 3. Hybrid distribution density vector is calculated by counting the number of points in each sector, from inner annular region to outer ones and within each annular region, in the angular order as discussed in Figure 2. The vector is: $(1,1,0,1,1,0,0,0,2,2,1,1,3$, $0,2,0,1,1,1,2,2,1,1,0,1,0,0,2,1,0,0,1)$.

Similar to the discussion in the annular case, the angular and hybrid densities can also be independently applied to define the color-space histogram matrix. These matrices are called as angular histogram matrix and hybrid histogram matrix respectively.

\section{Experiments and Comparisons}

Database Setup The test database consists of 500 art and real-world images divided into 41 similar groups such as scenery, human activities, animals, etc. All of the images are down-loaded from various web sites so that no prerestrictions such as camera type, brightness are specified for the testing.

Implementation Five techniques of calculating color histograms in the HVC color space are implemented: the traditional histogram, color coherent vector (CCV)[3] and three types of histogram described above: annular, angular, hybrid histogram matrices.

HVC color space is a non-linear transform from the raw RGB space. It is nothing else but the representation of the famous CIE 1976 $\left(L^{*} a^{*} b^{*}\right)[5$, Section 2.7.4] under the cylinder coordinate system. Explicitly, HVC is defined via $H=\arctan \left(\frac{b^{*}}{a^{*}}\right), V=L^{*}$, and $C=\sqrt{a^{* 2}+b^{*^{2}}}$

Feature vectors of the same dimensionality are generated for above mentioned five histogram. The dimensionality is set to 2048. The color space is quantized by making uniform partition in each color dimension. Furthermore, for 
the spatial color histograms, the parameters for quantizing color histogram sets under the polar coordinate $(r, \theta)$ are listed in Figure 4. Color coherent and incoherent vectors are set to be of dimension 1024 so that they contribute to final feature vectors of dimension 2048. Euclidean distance is used as the similarity measure for each case.

\begin{tabular}{||c|c|c|c|c|c||}
\hline Dim. & Ann. & Ang. & Hyb. & Trad. & $($ CCV $)$ \\
\hline $\mathrm{H}$ & 8 & 8 & 8 & 32 & 16 \\
$\mathrm{~V}$ & 4 & 4 & 4 & 8 & 8 \\
$\mathrm{C}$ & 4 & 4 & 4 & 8 & 8 \\
\hline $\mathrm{r}$ & 16 & 1 & 4 & 1 & 1 \\
$\theta$ & 1 & 16 & 4 & 1 & 1 \\
\hline Total & 2048 & 2048 & 2048 & 2048 & 2048 \\
\hline
\end{tabular}

Figure 4. Quantization parameters: the color and spatial granularity for 5 histograms.

Performance Comparison Figure 5 lists the average precision-recalls taken over 500 queries. The performance area[7] of each histogram is: 1415.74 (annular), 1352.65 (angular), 1314.20 (hybrid), 1156.77 (CCV) and 1037.22 (traditional), hence the improvement of the spatial color histograms over the traditional one are: $36.49 \%$ (annular), $30.41 \%$ (angular) and $26.71 \%$ (hybrid). The improvement of the spatial color histograms over CCV are: $22.39 \%$ (annular), $16.93 \%$ (angular) and $13.61 \%$ (hybrid).

As for the efficiency, the proposed techniques has the same time complexity as the traditional one: they are linear to the image size while CCV is not linear time. It is more efficient than $\mathrm{CCV}$ and correlogram[2].

\section{Conclusions}

The color spatial histograms can improve the performance of an image search engine based on color feature. Moreover, the approaches also provide a way to explore a particular range of color in more detail in the spatial domain so that it is extremely useful when a subset of colors is the main focus.

The idea of spatial densities can also be applied to the refinements of other image features as long as the spatial information is to be integrated into the feature.

\section{References}

[1] W. Hsu, T.S. Chua, and H. K. Pung. An integrated color-spatial approach to content-based image retrieval. ACM Multimedia Conference, pages 305 - 313, 1995.

[2] J. Huang. Color-Spatial Image Indexing and Applications. $\mathrm{PhD}$ thesis, Cornell Univ., 1998.

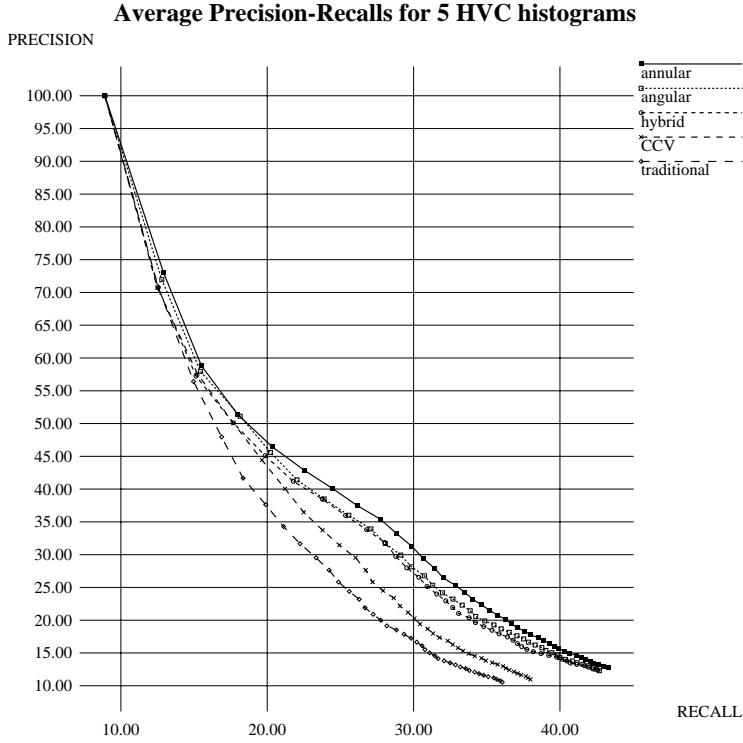

Figure 5. Performance comparison: Annular, angular and hybrid histograms outperform both the traditional histogram and color coherent vector. Number of retrieved images for each query: 40.

[3] G. Pass and R. Zabih. Histogram refinement for content-based image retrieval. IEEE Workshop on Applications of Computer Vision, pages 96-102, December 1996.

[4] R. Rickman and J. Stonham. Content-based image retrieval using color tuple histograms. SPIE proceedings, pages $2-7,1996$.

[5] J. Smith. Integrated Spatial and Feature Image Systems: Retrieval, Analysis and Compression. $\mathrm{PhD}$ thesis, Columbia Univ., 1997.

[6] J. Smith and S.-F. Chang. Tools and techniques for color image retrival. SPIE proceedings, pages $1630-$ 1639, 1996.

[7] R. K. Srihari, Z.F. Zhang, and A. Rao. Image background search: Combining object detection techniques with content-based image retrieval(cbir) systems. Proceedings of the IEEE Workshop on Content-Based Access of Image and Video Libraries (CBAIVL'99), in conjunction with CVPR'99, June 1999.

[8] M. Stricker and A. Dimai. Color indexing with weak spatial constraints. SPIE proceedings, 2670:29-40, February 1996.

[9] M. J. Swain and D. H. Ballard. Color indexing. International Journal of Computer Vision, 7(1):11-32, 1991. 